\title{
A rede social complexa de $O$ Senhor dos Anéis
}

The complex social network of The Lord of Rings

\author{
Mauricio Aparecido Ribeiro, Roberto Antonio Vosgerau, Maria Larissa Pereira Andruchiw, \\ Sandro Ely de Souza Pinto
}

Departamento de Física, Universidade Estadual de Ponta Grossa, Ponta Grossa, PR, Brasil

Recebido em 5 de agosto de 2015. Aceito em 28 de novembro de 2015

\begin{abstract}
Estudos de estruturas sociais vêm crescendo nos últimos anos, pois sua forma de compartilhamento e criação de conteúdo vêm atraindo o público em geral. Tais estruturas são observadas, por exemplo, em obras literárias. Um autor é J.R.R. Tolkien, com seus livros que descrevem um mundo fictício e seus habitantes. Estes livros trazem uma narrativa da criação da Terra-Média e toda a sua mitologia. Suas principais obras são: $O$ Silmarillion, O Hobbit e $O$ Senhor dos Anéis. O principal objetivo deste artigo é a análise da estrutura social emergente do conjunto destas obras, onde as relações sociais são descritas pelos critérios de alusão. compartilhamento de eventos e ligação direta, com as principais medidas de centralidade juntamente com a entropia estrutural de primeira ordem. Tirna-se então possível estabelcer uma analogia com o ensemble canônico da mecânica estatística, possibilitando analisar o grau de homogeneidade das ligações entre as comunidades formadas.
\end{abstract}

Palavras-chave: redes complexas, entropia, Tolkien.

Studies of social structures have been growing in the last years, because the form of sharing and creating contentes has attracted the genersl public. Such structures are observed, as an example, in literary works. A featured author is J.R.R. Tolkien, with his books that describe a fictional world and its inhabitants. These books bring a narrative of the creation of the Middle-Earth and all of its mythology. His main pieces are: The Silmarillion, The Hobbit, and The Lord of the Rings. The main objective of this article is the analysis of the social structures emerging of the conjunction of these works, in which the social relations are described by the reference criteria, shared events and direct bonds, with some major centrality measures together with the structural entropy of first order. It is then possible to establish an analogy with the canonic ensemble of statistical mechanics, with the possibility of analyzing the degree of homogeneity of the bonds between the communities.

Keywords: complex network, entropy, Tolkien.

\section{Introdução}

A teoria das redes complexas é amplamente utilizada para a modelagem de estruturas encontradas na natureza, denominadas de redes reais $[1$. Um exemplo de redes reais são as estruturas sociais, que são formadas pela interação de indivíduos [2]. Podemos identificar como exemplo de estrutura social, uma sala de aula, onde os alunos interagem nas mais diversas formas. A interação entre os indivíduos dáse o nome de relação social. Outro exemplo são comunidades de discussão na internet, como o Fa-

*Endereço de correspondência: mau.ap.ribeiro@gmail.com cebook, Twitter e outras que criam este vínculo social da interação de forma virtual, aumentando a possibilidade de interações entre pessoas [3, 4].

Porém, podemos observar que padrões do tipo social emergem de obras literárias, mais especificamente obras mitológicas. As obras de caráter mitológico apresentam interações entre o mundo real e o mundo dos seres fictícios, e foi demonstrado que estas redes são do tipo rede complexa [5 9]. Para a formação destas estruturas precisamos definir quais tipos de relações são formadas para que haja a interação social entre os indivíduos [10]. Definimos assim os seguintes critérios para as relações sociais: 
- Alusão à relação social: Quando em um diálogo as personagens descrevem um terceiro com o mínimo de detalhes em resposta das relações sociais implicadas.

- Compartilhamento de eventos: Quando as personagens estiverem no mesmo local e no mesmo instante, demonstrando participação da ação.

- Interação direta: Personagens que interagem diretamente.

Aqui, consideramos a declaração de uma relação social entre às personagens, como no trabalho pioneiro de Coleman 11.

Algumas obra de ficção baseada em várias mitologias e atos heroicos são as do escritor J.R.R. Tolkien 12. Estas obras relatam a criação da Terramédia e os eventos e feitos de seus habitantes. Esta obra de Tolkien se baseia em mitologia grega e escandinava, criando assim um mundo único. $\mathrm{O}$ mundo criado por Tolkien, denominado Arda, possui um início que é relatado na obra $O$ Silmarillion, onde o deus Ilúvatar com a sua sinfonia de criação com seus Ainur, vai arquitetando tudo. Outras obras como O Hobbit e o O Senhor dos Anéis, relatam feitos e batalhas épicas com seus respectivos heróis 13. Em $O$ Hobbit, são descritas as desventuras do hobbit Bilbo Bolseiro que viaja para Erebor com os anões para reconquistá-la de Smaug, e com essas desventuras, dando início aos acontecimentos de $O$ Senhor dos Aneis 14. A obra O Senhor dos Anéis é dividida em três partes, a primeira com $A$ Sociedade do Anel, que relata a comitiva formada por Elrond em Valfenda para auxiliar Frodo na destruição do Anel de poder. A segunda obra, As Duas Torres, relata o rompimento da Sociedade do Anel e também os acontecimentos que ocorrem nas torres de Orthanc, em Isengard, e Barad-dûr, em Mordor. A terceira obra, O Retorno do Rei, relata as batalhas de Gondor e de Rohan, e também a finalização da obra com a destruição do Anel de poder [15].

Estas obras de J.R.R. Tolkien influenciaram não somente a literatura, mas também jogos de RPG, cinema e a música.

Cada personagem de Tolkien possui uma árvore genealógica e carrega consigo toda a cultura de seu povo, bem como língua característica, familiares e relações sociais das mais diversas formas no decorrer do enredo.

O principal objetivo deste artigo é tratar uma rede social do ponto de vista interdisciplinar unindo literatura, matemática e física, também as medidas de centralidade de grau e sua distribuição, centralidade de intermediação, assortatividade, transitividade e a distância de caminho médio que são medidas clássicas na caracterização de redes. Também analisamos a formação de comunidades com o algoritmo de WalkTrap Comunities, que utiliza a caminhada aleatória. Também aplicamos a teoria do ensemble canônico, no qual, podemos cálcular a entropia estrutural. A entropia estrutural permite mensurar o grau de complexidade gerada pelas ligações que os vértices exercem com seus adjacentes.

\section{Resultados e discussões}

A Fig. 1 representa a rede social formada com as relações de alusão à relação social, compartilhamento de evento e ligação direta entre os personagens das obras de Tolkien. Esta estrutura apresentou 618 personagens (Vértices) e 19462 relações sociais (Arestas).

A distribuição de grau do tipo lei de potência com corte exponencial, dada pela Eq. (2) que permitiu corrigir o ajuste para vértices com alta probabilidade de conexão, onde ocorre uma anomalia, sendo caracterizada como os hubs de rede. Na estrutura social literária, são os vértices que representam as divindades e seres mitológicos, pois no decorrer dos relatos um grande número de personagens as conhecem. A Fig. 2 ilustra a distribuição de grau com o respectivo ajuste. A qualidade do ajuste foi é analisada pelo coeficiente de Person $\left(R^{2}=0,900672\right)$.

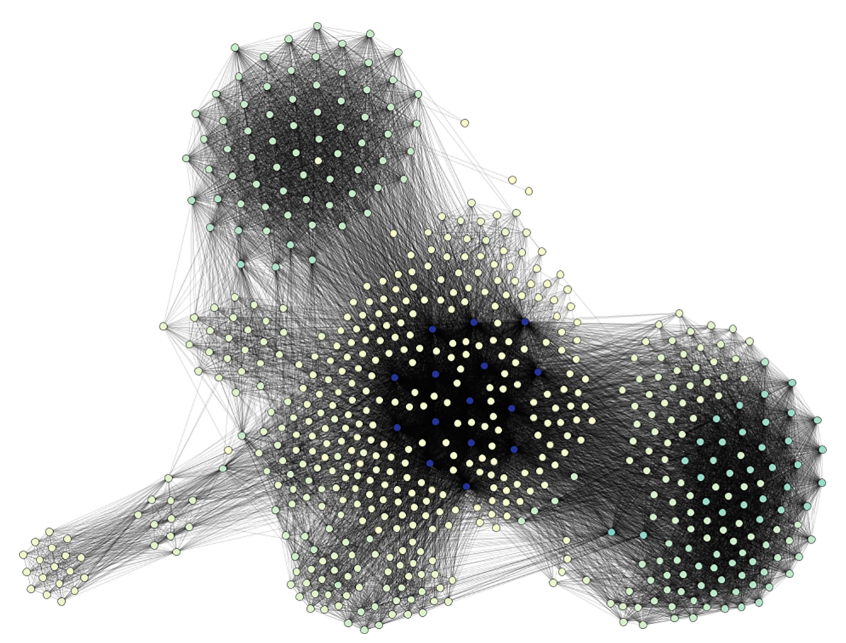

Figura 1: Rede social dos livros "O Silmarillion, O Hobbit e O Senhor dos Anéis vol. único". Com N = 618 (Personagens), $L=19462$ (Relações sociais). Os vértices de cor azul, são os vértices de maior conexão na rede. 
Com $\alpha=10^{-5}, \beta=-508,464, \gamma=0,07068 \mathrm{e}$ $\nu=0,06563$.

Na Tabela 1 estão apresentadas as medidas estruturais da rede formada com as divindades e sem as divindades.

A rede apresentou um caráter disassortativo, ou seja, personagens com maior conexão tendem a se conectarem com os de menor conexão. Isso acontece devido às obras terem uma descrição dos "deuses" (valar) da Terra-Média. A interação dos deuses com as personagens acontece de forma que a distância de caminho médio seja baixa. Isto evidencia o caráter onipresente da divindade, como demonstrado na Ref. [7. Na referência citada, quando às personagens mitológicas da rede são retiradas, a quantidade $D$ sobe de $\approx 2$ para $\approx 4.0$.

A medida de transitividade, que é referente aos triângulos formados na estrutura, indica a relação de compartilhamento de eventos e alusão, pois a transitividade calculada indica que os personagens tendem à formação de grupos coesos. A tendência de formação destes grupos coesos permitiu o cálculo

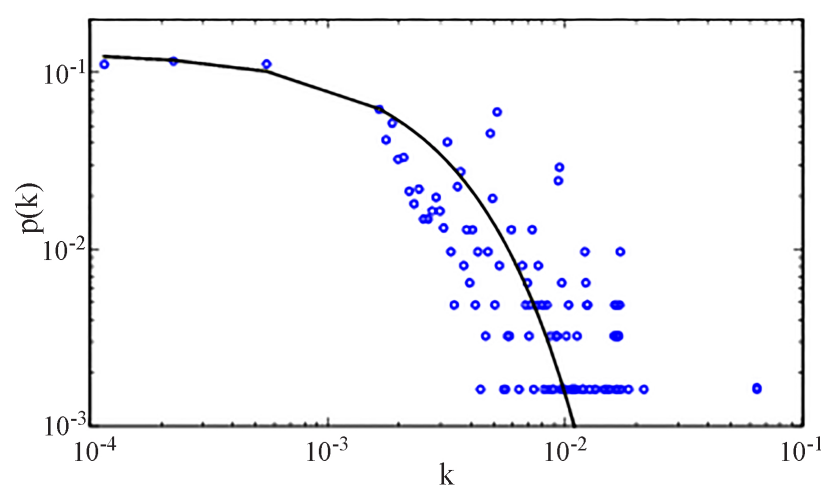

Figura 2: Distribuição de grau da estrutura social com seu respectivo ajuste dado pela equação 2, a qualidade do ajuste é dada pelo coeficiente de Pearson $R^{2}$. de comunidades na rede. Esta separação das comunidades ocorreu com a otimização da função de modularidade. A otimização se deu com o algoritmo de Walktrap Communities que utiliza a caminhada aleatória, identificando assim 5 comunidades como mostra a Fig. 3 .

Uma vez identificadas as comunidades da estrutura social, calculamos as mesmas medidas como mostra a Tabela 2 .

A Tabela 3 ilustra as medidas das propriedades estruturais e da entropia por comunidade. Após a retirada as divindades podemos observar a queda dos valores de entropia, como vemos também na comparação da Fig. 4 .

A Fig. 4 ilustra o comportamento das entropias calculadas com o formalismo da Eq. (10).

As comunidades apresentaram valores de transitividade altos, indicando alta densidade em ligações. Esta densidade é caracterizada pelo número de triângulos formados pela relação social de comparti-

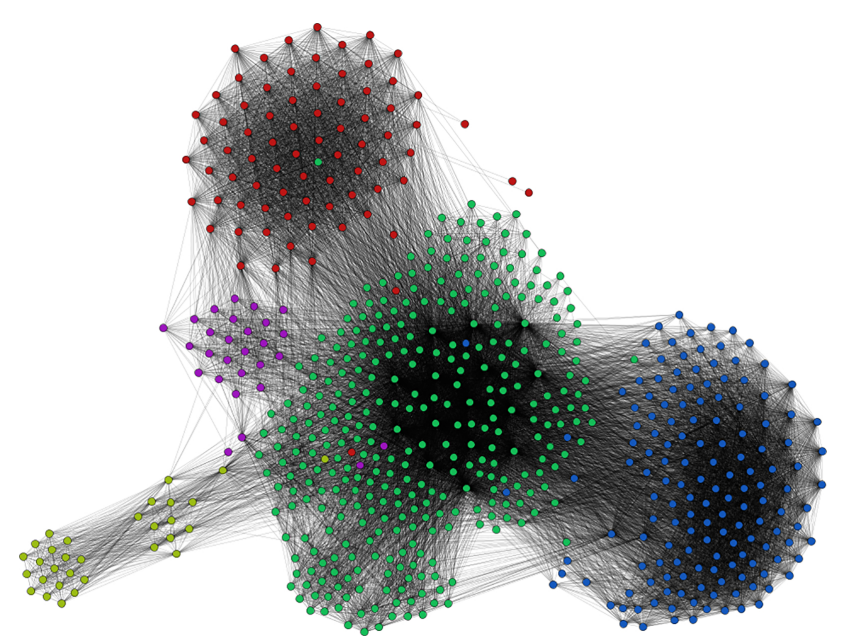

Figura 3: Rede social com as comunidades calculadas dos livros O Silmarillion, O Hobbit e O Senhor dos Anéis (vol. único).

Tabela 1: Valores das propriedades estruturais da rede social formada pelos personagens dos livros ( $O$ Silmarillion, $O$ Hobbit, $O$ Senhor dos Anéis, volume único) de J.R.R. Tolkien. Sendo $<k>$ o grau médio, $(T)$ a transitividade, $(A)$ a assortatividade, $(\langle B\rangle)$ centralidade de intermediação média, $(D)$ a distância de caminho médio, $(N)$ o número de vértices e $(E)$ o número de arestas.

\begin{tabular}{ccc}
\hline Propriedades & Estrutura Social Completa & Estrutura Social sem as divindades \\
\hline$<k>$ & 63 & 48 \\
$T$ & 0,318 & 0,377 \\
$A$ & $-0,367$ & $-0,378$ \\
$<B>$ & 304,894 & 307.816 \\
$D$ & 1,988 & 3,961 \\
$N$ & 618 & 608 \\
$E$ & 19462 & 14798 \\
\hline
\end{tabular}


Tabela 2: As propriedades estruturais das comunidades formadas pela rede social, sendo $<k>$ o grau médio, ( $T$ ) a transitividade, $(A)$ a assortatividade,$\langle B\rangle$ a centralidade de intermediação média, $(D)$ distancia de caminho médio, $(N)$ número de vértices e $(E)$ o número de arestas.

\begin{tabular}{ccccccccc}
\hline Comunidades & $\langle k\rangle$ & $T$ & $A$ & $\langle B\rangle$ & $D$ & $N$ & $E$ & $s_{1}$ \\
\hline 1 & 39 & 0,894 & $-0,5567$ & 67,47 & 1,896 & 331 & 6595 & 74,43 \\
2 & 64 & 0,958 & $-0,5355$ & 61,82 & 0,997 & 77 & 2494 & 24,94 \\
3 & 67 & 0,829 & $-0,496$ & 502,13 & 1,574 & 151 & 5105 & 83,85 \\
4 & 21 & 0,989 & $-0,4294$ & 22,27 & 1,201 & 28 & 305 & 25,49 \\
5 & 28 & 0,966 & $-0,3671$ & 362,93 & 1,062 & 31 & 436 & 9,68 \\
\hline
\end{tabular}

Tabela 3: As propriedades estruturais das comunidades formadas pela rede social com a extração das divindades, sendo $<k>$ o grau médio, $(T)$ a transitividade, $(A)$ a assortatividade,$\langle B\rangle$ a centralidade de intermediação média, ( $d$ ) distancia de caminho médio, $(N)$ número de vértices e $(E)$ o número de arestas.

\begin{tabular}{ccccccccc}
\hline Comunidades & $\langle k\rangle$ & $T$ & $A$ & $\langle B\rangle$ & $D$ & $N$ & $E$ & $s_{1}$ \\
\hline 1 & 65 & 0,657 & $-0,465$ & 42,161 & 1,569 & 149 & 5051 & 32,602 \\
2 & 66 & 0,997 & $-0,068$ & 0,906 & 1,062 & 32 & 436 & 8,134 \\
3 & 26 & 0,996 & $-0,027$ & 4,828 & 1,128 & 76 & 2493 & 14,621 \\
4 & 19 & 0,541 & $-0,071$ & 38,918 & 1,819 & 98 & 1306 & 23,249 \\
5 & 28 & 0,167 & $-0,566$ & 122,059 & 1,972 & 252 & 2419 & 8,435 \\
\hline
\end{tabular}

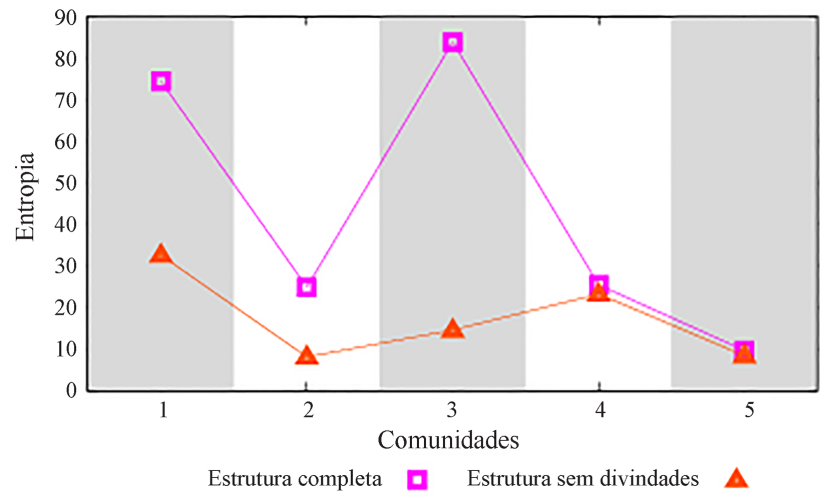

Figura 4: Gráfico comparativo das entropias estruturais considerando o vínculo da sequência de grau das comunidades formadas (subgrafos). Podemos observar o comportamento da entropia ao extrair as divindades da rede.

lhamento de evento e de alusão à relação social. No entanto, as comunidades apresentaram-se negativas quanto à assortatividade o que denota vértices de alta conexão ligando-se à vértices de baixa conexão e a presença de hubs na estrutura. Como podemos observar na comunidade 3 , dos deuses criadores e todos os membros ligados diretamente a eles. Esta comunidade apresentou maior grau médio e maior centralidade de intermediação média, isso ocorre pois a relação de alusão com as divindades e os outros habitantes da Terra-Média, aumenta a conectividade entre os personagens, exemplo é o elfo Elrond que está presente em todas as obras, sendo um dos vértices de intermédio entre a ação entre as personagens divinos e não divinos na alegoria Tolkeniana.
As personagens com esta carcteristica resultaram em heterogeneidade entre as ligações. Dentre estas comunidades, destaca-se a comunidade 3 apresentando-se como a mais heterogênea em ligações, pois a entropia estrutural calculada toma como vínculo a sequência de grau da estrutura social. Com isso a estrutura das obras de J.R.R. Tolkien é densa em ligações e suas comunidades são heterogêneas com o vínculo da sequência de grau. A distância de caminho médio da rede das comunidades mostrou que os percursos tomados na estrutura entre os personagens é no máximo 1,8, ou seja, um personagem se distância no mínimo a aproximadamente a duas ligações de outro personagem. Isto se dá pela pela a relação de alusão e compartilhamento de eventos, no enredo das obras isso é reforçado pelas guerras que ocorrem e os relatos sobre os antepassados, pois cada personagem de Tolkien possui uma história e uma genealogia.

\section{Metodologia}

Para a representação e análise matemática utilizaremos a teoria dos grafos. Define-se um grafo, como um par ordenado que relaciona um conjunto de vértice $(V)$ e um conjunto de $\operatorname{arestas}(E)$ [16, 17. As arestas podem ou não possuir uma direção, o que torna o grafo direcionado ou não direcionado, respectivamente. Esta forma de apresentação de uma rede social auxilia no cálculo das principais medidas 
de centralidade para caracterização das estruturas formadas.

\subsection{Centralidade de grau e sua distribuição}

A medida de centralidade de grau constitui do número de ligações que o vértice $i$ realiza com o vértice vizinho $j$, ou seja, é definido como o conjunto da cardinalidade do conjunto de vértices adjacentes a $i$. Desta medida, podemos extrair duas quantidades. A primeira o grau médio, que constitui da média aritmética da sequência de grau

$$
\langle k\rangle=\frac{\sum_{i=1}^{N} k_{i}}{N},
$$

sendo $N$ o número de vértices e o grau do vértice $i$. Outra medida é a distribuição de grau, sendo a fração de vértices com grau $k$. Esta distribuição possibilita de forma simples, quantificar o comportamento das ligações nas estruturas [18. O modelo proposto para o ajuste da distribuição de grau neste trabalho é dado por

$$
p(k \mid \alpha, \beta, \gamma, \nu)=\nu \frac{e^{-k \beta}}{(k+\alpha)^{\gamma}},
$$

sendo, $\gamma$ o parâmetro de decaimento, $\alpha$ o parâmetro de deslocamento, $\beta$ parâmetro do corte do fator exponencial e $\nu$ parâmetro de normalização da distribuição. Esta distribuição corrige a anomalia para vértices com alta probabilidade de conexão.

\subsection{Centralidade de intermediação}

A centralidade de intermediação é uma medida de influência do vértice $i$. Esta medida de centralidade é baseada nos menores caminhos, ou caminhos geodésicos que passam por $i[19$. Se $\sigma(i, j)$ é o número de caminhos geodésicos entre os vértices $i$ e $j$ e número destes caminhos que passam pelo vértice $l$ que intermedia os vértices $i$ e $j$, portanto a centralidade de intermediação pode ser definida como

$$
g_{l}=\frac{2}{(N-1)(N-2)} \sum_{i \neq j} \frac{\sigma_{l}(i, j)}{\sigma(i, j)} .
$$

\subsection{Coeficiente de aglomeração}

O coeficiente de aglomeração, ou transitividade, é uma medida que quantifica o número de triângulos na rede 20,21. Sendo calculada como

$$
C_{i}=\frac{2 n_{i}}{k_{i}\left(k_{i}-1\right)}
$$

Também podemos definir como a medida de probabilidade de um vértice $i$ estar conectado com um vértice adjacente $j$. Definimos então a transitividade média como

$$
\langle C\rangle=\frac{\sum C_{i}}{N} .
$$

\subsection{Assortatividade}

Esta medida é definida pela preferência da conexão entre os vértices. Este coeficiente pode assumir valores entre $[-1,1]$. No qual, valores positivos de $(A)$ indicam que os vértices de graus semelhantes estão conectados, caracterizando a rede como assortativa. Para valores negativos, vértices com grau elevado conectam-se com vértices com grau baixo, caracterizando assim a rede como disassortativa [22]. Esta grandeza é dada por

$$
A=\frac{\sum_{i} e_{i i}-\sum_{i} a_{i} b_{i}}{1-\sum_{i} a_{i} b_{i}}
$$

sendo $e_{i i}$ a fração de arestas que conectam os vértices $i$ e $j, a_{i}=\sum_{i} e_{i j}$ e $b_{j}=\sum_{j} e_{i j}$.

\subsection{Comunidades}

A estrutura de comunidades é definida como partições de um grafo $G$. Estas partições são obtidas com o cálculo da modularidade, dada por

$$
Q=\sum_{i}\left[e_{i i}-\left(\sum_{j} e_{i i}\right)^{2}\right]
$$

sendo $e_{i i}$ e $e_{i j}$ são as frações de arestas na rede que conectam no grupo $i$ deste grupo $j$. É a fração de arestas que se enquadram dentro das comunidades 23 .

\subsection{Distância média}

A distância de caminho médio de um grafo é a média das distâncias entre todos os pares de vértices. $\mathrm{Ou}$ seja, se $d(u, v)$ é a distância entre os vértices $u$ e $v$, $N$ o número de vértices do grafo, então

$$
D=\frac{\sum_{u, v \in V} d(u, v)}{\left(\begin{array}{c}
N \\
2
\end{array}\right)} .
$$




\subsection{Entropia estrutural}

A complexidade de uma estrutura de rede, depende da organização global que as ligações exercem com seus elementos. As redes reais apresentam diferentes níveis de organização, isso é devido a heterogeneidade das ligações entre os pares de vértices. Portanto, para mensurar o grau de organização, ou complexidade de uma rede, utilizamos a definição de entropia da mecânica estatística.

Esta entropia é calculada dado os vínculos estruturais das redes, como por exemplo a sequência de grau 24 25]. Este vínculo estrutural define o número de ligações que o vértice $i$ realiza com seus adjacentes. Neste caso, temos a função partição para este sistema, dada por

$$
Z_{1}=\sum_{\left\{a_{i j}\right\}} \prod_{i} \delta\left(k_{i}-\sum_{j} a_{i j}\right) \exp \left[\sum_{i<j} h_{i j} a_{i j}\right]
$$

A aproximação para entropia deste ensemble é dada pelo método dos multiplicadores de Lagrange para otimização da Eq. (9), portanto, a entropia de ordem um $\left(s_{1}=\frac{S_{1}}{N}\right)$ por vértice da rede é dada por

$$
s_{1} \approx-\frac{1}{N}\left[\sum k_{i}\left(\ln \left(k_{i}\right)-1\right)-\frac{1}{2} \sum_{i} \ln \left(2 \pi k_{i}\right)+\frac{1}{2}\langle k\rangle N[\ln (\langle k\rangle N)-1]-\frac{1}{2}\left(\frac{\left\langle k^{2}\right\rangle}{\langle k\rangle}\right)^{2}\right],
$$

sendo $N$ o número de vértices da rede, $k_{i}$ é o grau do vertice $i,\langle k\rangle$ é o grau médio.

\section{Formação das redes}

Para a formação da rede social literária foram utilizadas as obras $O$ Silmarillion, O Hobbit e $O$ Senhor dos Anéis, vol. único, do escritor J.R.R. Tolkien. A mineração de personagens das obras foi considerando as restrições sociais de alusão, compartilhamento de evento e interação direta, as quais formaram as ligações entre os personagens. Esta divisão para a mineração é dada em três partes.

1. Selecionar no texto os personagens que estão participando do enredo da obra;

2. Análise do tipo de conexão dado por diálogos, eventos e descrição. Estas conexões devem respeitar as restrições de alusãom compartilhamento de evento e interação direta;

3. Formação do padrão tipo rede social dado os passos 1 e 2 .

A Fig. 5 mostra a mineração dos personagens em um trecho do livro A Sociedade do Anel que faz parte do conselho de Elrond.

\section{Conclusão}

O objetivo deste artigo foi construir uma rede social das obras O Senhor Dos Anéis, O Silmarillion e $O$ Hobbit. A rede social apresentou-se disassortativa, ou seja, vértices com alta conexão se relacionam com vértices de baixa conectividade e com transitivi-

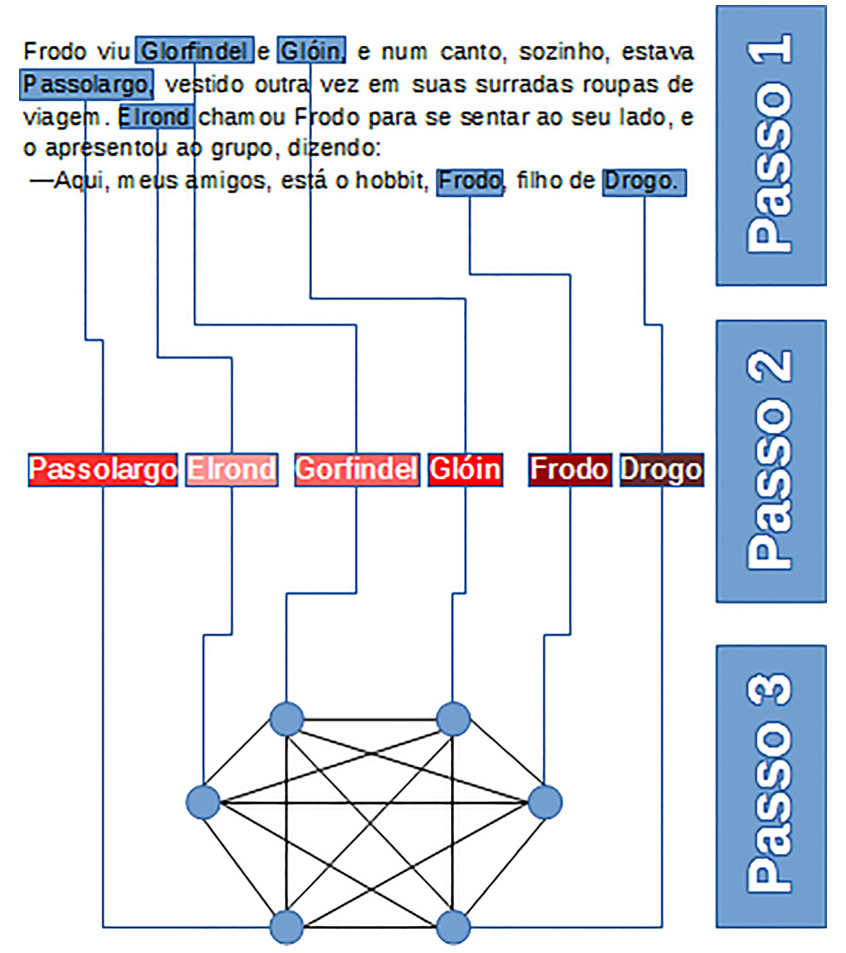

Figura 5: Esquema de mineração dos personagens de um trecho da obra literária $A$ sociedade dos Anel. O Passo 1 a identificação no texto dos personagens. No passo 2, os nomes dos personagens são separados e no passo 3 são analisadas as três relações sociais que serão as ligações entre os personagens, formando assim a rede social literária. 
dade de aproximadamente 0,30. Esta transitividade reflete na formação de triângulos na rede, caracterizando sua densidade. A distribuição de grau da rede comportou-se como uma distribuição do tipo lei de potência com truncamento exponencial, como dado na Eq. (2). O ajuste foi satisfatório como mostra o coeficiente de Pearson. Nesta rede foram encontradas 5 comunidades com o algoritmo de Walktrap Communities, que otimiza a função de modularidade por caminhada aleatória. A estatística destas comunidades mostrou que a comunidade 2 possui uma centralidade de intermediação média maior, isso acontece em virtude das relações de alusão à relação de amizade e compartilhamento de eventos que os seres divinos tem no enredo das obras.

Alta densidade de conexões como apresentado na medida de transitividade, é as custas das relações de compartilhamento de eventos e ligação direta. Todas as comunidades possuem um caráter disassortativo como verificado na estrutura como um todo. Com o cálculo da entropia estrutural podemos quantificar a complexidade das estruturas formadas pelas comunidades, na qual se destaca mais a comunidade 3 , onde se apresentam os seres divinos e criaturas mitoglógias próximas a eles.

A estrutura emergente das obras de Tolkien apresentou um caráter disassortativo, transitivo e com uma distribuição de grau com caracteristicas de redes sociais.

A interação dos deuses com as personagens aconteceram de forma que a distância de caminho médio possuindo $D=1,988$, com a extração das divindades a medida passa para $D=3,961$, que segundo 7 evidência o caráter onipresente das divindades e diminuindo assim a entropia estrutural.

\section{Agradecimentos}

GDNLSC agradece a CAPES pela bolsa disponível durante o programa de doutorado. Os autores também agradecem ao árbitro pelos pareceres e sugestões dadas para tornar este trabalho melhor enquadrado ao público alvo.

\section{Referências}

[1] A. Albert and R. Barabasi, Rev. Mod. Phys 74, (2002).

[2] S.H. Strogatz, Nat 410, 4603 (2001).

[3] H. Jo and H.Y. Eom, Sci. Rep 4, 5757, (2014).

[4] G. Kossinets and D.J. Watts, Sci. 311, (2006).
[5] R. Carron and P.M. Kenna, EPL 99, 2 (2012).

[6] R. Carron and P.M. Kenna, Eur. Phys. J. B 86, 407(2013).

[7] P.J. Miranda, M.S. Baptista and S.E.S. Pinto, arXiv:1306.2537 (2013).

[8] A.C. Sparavigna, I. J. Sci. 2, 10 (2013).

[9] J. Rossello, F. Alberich and R. Miro-Julia, arXiv0202174 02, (2002).

[10] C.F. Linton, Soc. Net 1 (1979).

[11] J.S. Coleman, The Adolescent Society (Free Press, New York, 1961).

[12] J. Campbell, The Hero With a Thousand Faces (Princiton University Press, Princiton, 1949).

[13] J.R.R. Tolkien, O Silmarillion (WMF Martins Fontes, 2011), 5th ed.

[14] J.R.R. Tolkien, O Hobbit (WMF Martins Fontes, 2012), 5th ed.

[15] J.J.R. Tolkien, O Senhor dos Aneis( WMF Martins Fontes, 2001), 5th ed.

[16] M. Newman, S. Rev 45, 2 (2003).

[17] M.E.J. Newman, Phy Rev E, 67, 026126 (2013).

[18] F.N. Silva, M.P. Viana, B.A.N. Travencolo and L.F. Costa, J. Info 5, 3 (2011).

[19] B. Ulrik, J, Math Soc 25, 2 (2001).

[20] M.P. Satorras, R. Barrat, A. Barthelemy and A. Vespignani, Proc Natl Acad Sci USA. 11, 1 (2004).

[21] B. Stefano, L. Vito, M. Yamir, C. Martin and D.U. Hwang, Phy Rep 4, 424, (2006).

[22] M.E.J. Newman, Phy, Rev. Let 89, 20 (2002).

[23] P. Janette, G. Kaberi, E. Lawrie and R. Raeside, JCN 21, 19 (2012).

[24] G. Bianconi, EPL 81, 2 (2008).

[25] G. Bianconi, Phys. Rev. E 79, 3 (2009). 